\section{Summary}

Insulin-mimetic effects of vanadate and pervanadate, potential therapeutic use in diabctes mellitus

To fully understand the pathophysiology of diabetes mellitus, the elucidation of the mechanism of insulin action is essential. It is known that insulin binds to its cell surface membrane receptor, composed of $2 \alpha^{-}$, externally oriented, and $2 \beta^{-}$, transmembrane and cytoplasmic, subunits held together by disulfide bonds. The binding of insulin stimulates an autophosphorylation reaction of tyrosine residucs of the $\beta$-subunit resulting in an activated receptor tyrosinc kinase. The importance of this kinase in transducing insulin's signal was tested in our laboratory using the insulin-mimetic agents, vanadate, a known inhibitor of phosphotyrosyl phosphatase and hydrogen peroxide $\left(\mathrm{H}_{2} \mathrm{O}_{2}\right)$. During these studies, we discovered that a mixture of vanadate plus $\mathrm{H}_{2} \mathrm{O}_{2}$ had a powerful synergistic effect to mimic many of insulin's biological actions in rat adipocytes. Further studies revea-

(Suite p. 256)

\footnotetext{
ADRESSE

I.G. Fantus : chef du centre de mélabolisme de l'hôpital Royal Vicloria, direcleur associé. G. Deragon. B.I. Posner : chef du Service d'endocrinologie de l'hôpilal Royal Vicloria, direcleur. Protein and polypeptide hormone laboratory, Rm 2-11, Strathiona medical Bld, McGill university, 3640 rue Université, Montréal, Québec, H3A 282 Canada.
}

\title{
Effet insulinomimétique du vanadate et du pervanadate : potentiel thérapeutique dans le diabète sucré
}

\author{
I. Georges Fantus, Guy Deragon, \\ Barry I. Posner
}

I 1 semble évident aujourd'hui que le diabète sucré, et particulièrement le diabète non insulinodépendant de type II, est caractérisé par une résistance des tissus cibles à l'action de l'insuline, d'où les efforts accrus pour comprendre le mécanisme d'action de l'insuline et les implications cliniques qui peuvent en résulter.

Au cours des vingt dernières années, il a été bien établi que la première étape de l'action de l'insuline est la liaison de l'hormone à son récepteur membranaire spécifique. Par ailleurs, le clonage de l'ADNc et l'analyse de la séquence des acides aminés du récepteur ont révélé que ce dernier est composé de 2 sous-unités $\alpha$ et de 2 sous-unités $\beta$ qui forment une structure tétramérique maintenue par des ponts disulfures [1-3]. L'insuline se lie à la partie $\alpha$ du récepteur qui est située du côté externe de la paroi cellulaire : cette liaison produit une autophosphorylation de la sous-unité $\beta$ qui est située du côté interne de la paroi cellulaire (voir P. Freychet, $\mathrm{m} / \mathrm{s}$ $n^{\circ} 2$, vol. 4, p. 70 et E. Clauser, même numéro, p. 72 pour une revue du mécanisme d'action de l'insuline). Cette autophosphorylation résulterait d'une diminution de l'influence inhibitrice de la sous-unité $\alpha$, et impliquerait un transfert de phosphates de l'ATP vers un ensemble de résidus tyrosyls. La sous-unité $\beta$ requiert la phosphoryla- tion d'au moins trois résidus tyrosyls $(1146,1150$ et 1151) pour exercer au maximum son activité tyrosine kinase et permettre la phosphorylation de substrats exogènes [4]. Cette observation a fait postuler que la transduction du signal nécessite la phosphorylation de résidus tyrosyls de protéines cellulaires endogènes capables de modifier le transport membranaire et les activités enzymatiques associées aux effets biologiques de l'insuline [5]. L'identification de ces substrats protéiques fait aujourd'hui l'objet de recherches intensives dans plusieurs laboratoires.

L'une des approches employées dans l'étude du mécanisme d'action de l'insuline est l'utilisation d'agents insulinomimétiques. La connaissance de leurs mécanismes d'action et la corrélation de leurs effets biologiques à ceux de l'insuline ont permis de mettre en évidence le rôle de l'autophosphorylation et l'importance de l'activité kinasique, en tant qu'événements critiques dans la médiation des actions insuliniques [5]. C'est ainsi que nous avons utilisé le vanadate $[6,7]$, un inhibiteur connu de la phosphotyrosyl phosphatase [8]. Les effets insulinomimétiques des dérivés du vanadium (orthovanadate de sodium, sulfate de vanadyl) sont bien documentés depuis qu'en 1980 l'on démontrait leur capacité à stimuler l'oxydation du glucose dans les 


\section{Summary (suite)}

led that a novel compound, a pcroxide of vanadate which we have termed pervanadate, was generated in this mixture. Pervanadate activated the insulin receptor kinase in intact adipocytes and was 100-1 000 times more potent than vanadate. This activation was associated with enhanced tyrosine phosphorylation of the $\beta$ subunit. It had no action on partially purified receptor preparations. Investigations into its mechanism revealed that although pervanadate weakly inhibited alkaline phosphatase, it was a powerful inhibitor of a rat hepatocyte phosphotyrosyl phosphatase. Vanadate itself has been administered orally in a number of studics to treat diabetes mellitus in rodents. Whether pervanadate will be a useful agent will depend on the development of a stable compound and testing its efficacy and possible toxic effects. At the very lcast, the discovery of a potent phosphotyrosyl phosphatase inhibitor will be a useful probe into the biochemistry of insulin action and the role of these phosphatases in cell biology. adipocytes de rat $[9,10]$. En fait, un an plus tôt, Tolman présentait des résultats qui suggéraient déjà que le vanadate pouvait mimer l'insuline au niveau des trois principaux tissus cibles: adipocytes, muscle et foie [11]. Depuis lors, plusieurs chercheurs ont confirmé ces observations et les ont étendues à d'autres tissus, de même qu'à des systèmes de cellules en culture [12-18].

Le mécanisme d'action du vanadate semble nécessiter l'activité de la tyrosine kinase du récepteur de l'insuline. Quoique certains aient suggéré une activation directe [12], la plupart des chercheurs sont d'accord pour dire

Tableau I

EFFETS INSULINOMIMÉTIQUES DU VANADATE ET DU PERVANADATE

\begin{tabular}{|c|c|c|c|}
\hline & Vanadate & Pervanadate & Références \\
\hline $\begin{array}{l}\text { Adipocytes } \\
\text { Transport du glucose } \\
\text { Oxydation du glucose } \\
\text { Liaison IGF-2 } \\
\text { Liaison LDL } \\
\text { Lipogenèse } \\
\text { Glycogène synthétase } \\
\text { Lipolyse }{ }^{* *} \\
\mathrm{Cl}^{2}+\mathrm{Mg}^{2}+\text { ) ATPase ** } \\
\text { Phosphorylation des résidus tyrosyls } \\
\text { du récepteur de l'insuline } \\
\text { Activité tyrosine kinase du récepteur } \\
\text { de l'insuline } \\
\text { Synthèse des protéines } \\
\text { Modulation de la concentration des } \\
\text { récepteurs insuliniques de surface } \\
\text { cellulaire }\end{array}$ & $\begin{array}{l}+ \\
+ \\
+ \\
+ \\
+ \\
+ \\
+ \\
+ \\
+ \\
+ \\
+ \\
+\end{array}$ & $\begin{array}{l}+ \\
\text { ND } \\
+ \\
\text { ND } \\
+ \\
\text { ND } \\
+ \\
\text { ND } \\
+ \\
+ \\
+ \\
+\end{array}$ & $\begin{array}{l}{[10]} \\
{[9,10,11]} \\
{[7]} \\
{[28]} \\
{[20]} \\
{[12]} \\
{[20]} \\
{[13]} \\
{[12,20]} \\
{[7,12,20]} \\
{[20]} \\
{[14]}\end{array}$ \\
\hline $\begin{array}{l}\text { Muscle squelettique } \\
\text { Transport du glucose } \\
\text { Glycolyse } \\
\text { Synthèse du glycogène } \\
\text { Oxydation du glucose } \\
\text { Synthèse des protéines }\end{array}$ & $\begin{array}{l}+ \\
+ \\
+ \\
+ \\
-\end{array}$ & $\begin{array}{l}\text { ND } \\
\text { ND } \\
\text { ND } \\
\text { ND } \\
\text { ND }\end{array}$ & $\begin{array}{l}{[15]} \\
{[15]} \\
{[15]} \\
{[15]} \\
{[15]}\end{array}$ \\
\hline $\begin{array}{l}\text { Hépatocytes/Cellules d'hépatome } \\
\text { Synthèse du glycogène } \\
\text { Sécrétion d'apolipoprotéine } 8^{* *} \\
\text { Lipogenèse } \\
\text { Activité tyrosine kinase du récepteur } \\
\text { de l'insuline } \\
\text { Activité phosphotyrosyl phospha- } \\
\text { tase ** }\end{array}$ & $\begin{array}{l}+ \\
+ \\
+ \\
+{ }^{*} \\
+{ }^{*}\end{array}$ & $\begin{array}{l}\text { ND } \\
\text { ND } \\
\text { ND } \\
+{ }^{*} \\
+{ }^{*}\end{array}$ & $\begin{array}{l}{[16]} \\
{[16]} \\
{[16]}\end{array}$ \\
\hline $\begin{array}{l}\text { Autres cellules } \\
\text { Mitogenèse }\end{array}$ & + & ND & {$[17,18]$} \\
\hline
\end{tabular}

ND : non déterminé. * Fantus I.G. et Posner B.I., données non publiées. * inhibition.

rect du vanadate via son action inhibitrice sur la phosphotyrosyl phosphatase. L'activité tyrosine kinase serait donc maintenue ou augmentée selon le degré d'autophosphorylation des résidus tyrosyls du récepteur de l'insuline.

Dans notre laboratoire, alors que nous étudions les effets du vanadate et du peroxyde d'hydrogène $\left(\mathrm{H}_{2} \mathrm{O}_{2}\right)$, autre agent insulinomimétique qui stimule la phosphorylation des résidus tyrosyls du récepteur de l'insuline [19], nous avons observé qu'un mélange de ces deux agents possédait une activité insulinomimétique très puissante [7]. En effet, lorsque des adipocytes intacts de rat sont expo- sés à ce mélange, nous observons une forte phosphorylation des résidus tyrosyls du récepteur de l'insuline, accompagnée d'une activité tyrosine kinase augmentée, mesurable à l'aide du substrat artificiel Poly (Glu : Tyr, $4: 1)[7,20]$. Nous avons également observé que si l'on exposait le mélange à la catalase, une enzyme qui dégrade le $\mathrm{H}_{2} \mathrm{O}_{2}$, l'effet insulinomimétique était conservé [21] : cela suggère que c'est la formation d'un nouveau composé, un peroxyde de vanadate, qui est responsable de l'effet du mélange, et non la simple action synergique du vanadate et du $\mathrm{H}_{2} \mathrm{O}_{2}$. Ce nouvel agent, que nous avons appelé "pervanadate ", est ins- 


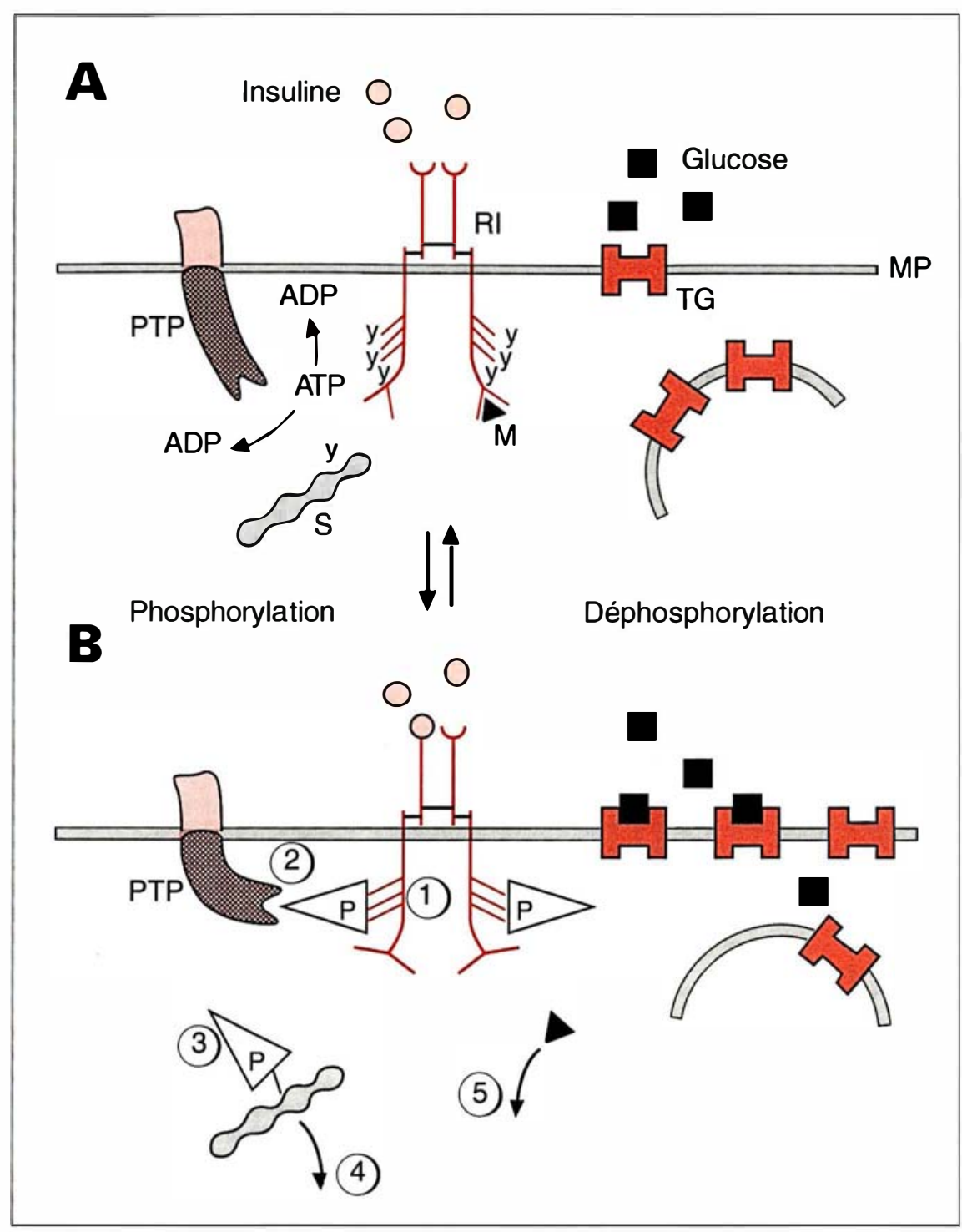

Figure 1. A. Mécanismes d'action possible du vanadate et du pervanadate. Après síimulation par l'insuline, le récepteur de l'insuline (RI), composé de ses sous-unités $\alpha$ et $\beta$, subit une autophosphorylation de ses résidus tyrosines (Y). Le récepteur est ainsi transformé en une tyrosine protéine kinase qui phosphoryle certains substrats (S) protéiques endogènes sur des tyrosines, modifiant ainsi leur fonction. Ces substrats pourraient eux-mêmes être des enzymes. L'autophosphorylation change également la conformation du récepteur et les interactions possibles avec d'autres messagers (M) (?) protéiques. Ce sont ces substrats phosphorylés et/ou ces messagers qui seraient les intermédiaires des multiples effets de l'insuline. "I existe vraisemblablement un niveau de base constant d'autophosphorylation ; l'activité kinase du récepteur est donc maintenue à son état basal par une phosphotyrosyl phosphatase (PTP) qui déphosphoryle continuellement le RI et peut-être ses substrats. B. Les mécanismes d'action possibles du vanadate et du pervanadate sont: une action directe, par activation de la tyrosine kinase du RI (1) ou par inhibition d'une PTP transmembranaire et/ou cytoplasmique qui déphosphoryle le RI (2) et/ou ses substrats (3) une action plus distale se situant en aval d'une enzyme phosphorylée sur une tyrosine (4) ou d'un messager transducteur de signal (5). TG : transporteur de glucose; ATP: adénosine triphosphate; $P$ : phosphate; MP: membrane plasmique. table et perd son activité au bout de 2 à 3 heures en l'absence d'un excès de $\mathrm{H}_{2} \mathrm{O}_{2}$. La nature précise du composé actif est inconnue pour le moment, plusieurs types de peroxydes de vanadium pouvant être produits. Nous avons, en outre, démontré que le pervanadate stimule la lipogenèse, le transport du glucose, la liaison du facteur de croissance IGFII à son récepteur et qu'il inhibe la lipolyse (Tableau I). Fait intéressant, le pervanadate stimule également la synthèse des protéines - alors que le vanadate est sans effet [15] — et il s'avère dans tous les cas être de 100 à 1000 fois plus puissant que le vanadate [7]. Cela est dû à une entrée en action très rapide, de même qu'à une activité intrinsèque plus importante (résultats non publiés).

En étudiant son mécanisme d'action, nous avons remarqué que le pervanadate était incapable de stimuler l'autophosphorylation ou l'activité tyrosine kinase du récepteur insulinique partiellement purifié [20, 21]. D'autre part, l'effet du vanadate sur l'activité phosphatase étant connu, nous avons testé l'action du pervanadate sur cette activité. Contrairement au vanadate, le pervanadate n'inhibe que faiblement l'activité déphosphorylante de la phosphatase alcaline; en revanche, il inhibe fortement la phosphotyrosyl phosphatase microsomiale extraite du foie de rat [20]. L'ensemble de ces résultats suggère donc que l'action insulinomimétique du pervanadate est principalement due à un pouvoir inhibiteur puissant sur une phosphotyrosyl phosphatase spécifique. Des données préliminaires indiquent que ce nouvel agent mime également l'insuline au niveau du tissu hépatique (Tableau I). Par ailleurs, des études récentes réalisées par Zick [22] indiquent que le pervanadate stimule la phosphorylation de résidus tyrosyls de protéines endogènes dans les cellules d'hépatome de rats $\mathrm{Fao}$; ces protéines seraient des substrats de la kinase du récepteur de l'insuline.

En 1985, Heyliger et ses collègues rapportaient que l'administration orale de vanadate à des rats diabétiques pouvait normaliser leur glycémie [23]. Ce qui fut par la suite confirmé par plusieurs autres grou- 
pes [24-26]. Le vanadate est également connu pour sa capacité à améliorer la sensibilité de certains tissus à l'insuline [24] et à corriger tant des activités enzymatiques anormales que des concentrations inappropriées de métabolites intracellulaires [25]. Bien que le vanadate puisse mimer plusieurs effets d'une thérapie à l'insuline, il a été observé à maintes reprises que les animaux ainsi traités présentaient une croissance retardée. Cela suggère soit que les effets anaboliques de l'insuline sont peu ou mal reproduits, soit que le vanadate est par lui-même toxique. Une telle toxicité pourrait être liée à l'inhibition par le vanadate de plusieurs ATPases impliquées dans les canaux ioniques phosphorylés [27].

Puisque, d'une part, le pervanadate mime l'insuline à des concentrations beaucoup plus faibles et que, d'autre part, ce composé stimule la synthèse protéique, il serait très intéressant d'observer ses effets dans le traitement du diabète animal. Une telle étude nécessiterait toutefois l'identification et la synthèse d'une molécule stable qui puisse être facilement administrée. Selon des données récentes obtenues dans notre laboratoire avec des modèles in vitro de résistance à l'insuline, des adipocytes insensibles à l'insuline répondent au vanadate et au pervanadate (données non publiées). Ces résultats sont confirmés par des études in vivo chez la souris insulinorésistante ob/ob [28]. Il reste maintenant à déterminer si ces observations sont liées à l'activité phosphotyrosyl phosphatase et quelle est l'importance de cette activité dans le mécanisme d'action de l'insuline. Enfin, que le pervanadate s'avère utile ou non dans le traitement du diabète, il est un outil puissant qui devrait permettre de mieux comprendre le mécanisme d'action de l'insuline et la pathogénie de la résistance à l'insuline

\section{RÉFÉRENCES}

1. Czech MP. The nature and regulation of the insulin receptor. Ann Rev Physiol $1984 ; 47: 357-81$

2. Rosen OM. After insulin binds. Science
3. Kahn CR, White MF. The insulin receptor and the molecular mechanism of insulin action. J Clin Invest 1988; 82 : 1151-6.

4. Czech MP, Klarlund JK, Yagaloff KA, Bradford AP, Lewis RE. Insulin receptor signaling. Activation of multiple serine kinases. J Biol Chem 1988 ; 263 : 11017-20

5. Gammeltoft S, Van Obberghen E. Protein kinase activity of the insulin receptor Biochem J 1986 ; 235 : 1-11.

6. Kadota S, Fantus IG, Hersh B, Posner BI. Vanadate stimulation of IGF binding to rat adipocytes. Biochem Biophys Res Commun $1986 ; 138: 174-8$.

7. Kadota S, Fantus IG, Deragon G, Hersh B, Guyda HJ, Posner BI. Stimulation of IGF-II receptor binding and insulin receptor kinase activity in rat adipocytes : effects of vanadate and $\mathrm{H}_{2} \mathrm{O}_{2} . J$ Biol Chem 1987；262 : 8252-6.

8. Swarup G, Cohen S, Garbers DL. Inhibition of membrane phosphotyrosyl-protein phosphatase activity by vanadate. Biochem Biophys Res Commun 1982 ; 107 : 1104-9.

9. Dubyak GR, Kleinzeller A. The insulin mimetic effects of vanadate in isolated rat adipocytes. Dissociation from effects of vanadate as a $\left(\mathrm{Na}^{+}-\mathrm{K}^{+}\right)$ATPase inhibitor. J Biol Chem 1980 ; 255 : 5306-12.

10. Shechter Y, Karlish SJD. Insulin-like stimulation of glucose oxidation in rat adipocytes by vanadyl (IV) ions. Nature 1980 ; 284: 556-8

11. Tolman EL, Barris I, Burns M, Pansini A, Partridge R. Effects of vanadium on glucose metabolism in vitro. Life Sciences $1979 ; 25: 1159-64$

12. Tamura S, Brown TA, Whipple JH, et al. A novel mechanism for the insulin-like effect of vanadate on glycogen synthase in rat adipocytes. J Biol Chem 1984; 259 : 6650-8.

13. Delfert DM, McDonald JM. Vanadyl and vanadate inhibit $\mathrm{Ca}^{2}+$ transport systems of the adipocyte plasma membrane and endoplasmic reticulum. Arch Biochem Biophys 1985 ; 241 : 665-72.

14. Marshall S, Monzon R. Downregulation of cell surface insulin receptors in primary cultured rat adipocytes by sodium vanadate. Endocrinology $1987 ; 121$ : 1116-22.

15. Clark AS, Fagan JM, Mitch WE. Selectivity of the insulin-like actions of vanadate on glucose and protein metabolism in skeletal muscle. Biochem J 1985; 232 : 273-6.

16. Jackson TK, Salhanick AI, Sparks JD, Sparks CE, Bolognino M, Amatruda JM Insulin-mimetic effects of vanadate in primary cultures of rat hepatocytes. Diabetes $1988 ; 37: 1234-40$

17. Hori C, Oka T. Vanadate enhances the stimulatory action of insulin on DNA synthesis in cultured mouse mammary gland. Biochem Biophys Acta 1980 ; 610 : 235-40.

18. Smith JB. Vanadium ions stimulate DNA synthesis in Swiss mouse 3T3 and
3T6 cells. Proc Natl Acad Si USA 1983 : 80 : 6162-66.

19. Hayes CR, Lockwood DH. Role of insulin receptor phosphorylation in the insulinomimetic action of hydrogen peroxide Proc Natl Acad Sci USA 1987 ; 84 : 8115-9.

20. Fantus IG, Kadota S, Deragon G., Foster B, Posner BI. Pervanadate (peroxides of vanadate) micims insulin action in rat adipocytes via activation of the insulin receptor tyrosine kinase. Biochemistry 1989 ; $28: 8864-71$

21. Kadota S, Fantus IG, Deragon G, Guyda HJ, Hersh B, Posner BI. Peroxides of vanadium : a novel and potent insulinmimetic agent which activates the insulin receptor kinase. Biochem Biophys Res Commun $1987 ; 147: 259-66$

22. Heffetz D, Bushkin I, Dror R, Zick Y. The insulinomimetic agents $\mathrm{H}_{2} \mathrm{O}_{2}$ and vanadate stimulate protein tyrosine phosphorylation in intact cells. J Biol Chem 1990 ; 265 : 2896-902.

23. Heyliger CE, Tahiliani AG, McNeill $\mathrm{JH}$. Effect of vanadate on elevated blood glucose and depressed cardiac performance of diabetic rats. Science $1985 ; 227: 1474-7$.

24. Meyerovitch J, Farfel Z, Sack J, Shechter $\mathrm{Y}$. Oral administration of vanadate normalizes blood glucose levels in streptozotocin-treated rats. $J$ Biol Chem 1987 ; 262: 6658-62.

25. Gil J, Miralpeix M, Carreras J, Bartrons R. Insulin-like effects of vanadate on glucokinase activity and fructose 2,6 bisphosphate levels in the liver of diabetic rats. J Biol Chem 1988 ; 263 : 1868-71.

26. Bendayan M, Gingras D. Effects of vanadate administration on blood glucose and insulin levels as well as on the exocrine pancreatic function in streptozotocin-diabetic rats. Diabetologia 1989 : 32 : 561-7.

27. Pedersen PL, Carafoli E. Ion motive ATPases. I. Ubiquity, properties and significance to cell function. Trends Biochem $S a$ $1987 ; 12$ : 146-50.

28. Meyerovitch J, Rotenberg P, BonnerWeir S, Kahn CR, Schechter Y. Vanadate normalizes hyperglycemia in two models of non insulin-dependent diabetes mellitus (soumis pour publication).

\section{TIRÉS A PART}

I. G. Fantus.

\section{Remerciements}

Supported by grants from the Medical Research Council (Canada) to I.G.F. and B.I.P. I.G.F. est un chercheur boursier du Fonds de Recherche en Santé du Québec. 